\title{
Lifetime Prediction Routing in Mobile Ad Hoc Networks
}

\author{
Morteza Maleki, Karthik Dantu, and Massoud Pedram \\ Dept. of EE-Systems, University of Southern California, Los Angeles, CA, 90089 \\ \{morteza, dantu, massoud\}@ sahand.usc.edu
}

\begin{abstract}
One of the main design constraints in mobile adhoc networks (MANETs) is that they are power constrained. Hence, every effort is to be channeled towards reducing power. More precisely, network lifetime is a key design metric in MANETs. Since every node has to perform the functions of a router, if some nodes die early due to lack of energy, it will not be possible for other nodes to communicate with each other. Hence, the network will get disconnected and the network lifetime will be adversely affected. This paper presents a lifetime prediction routing protocol for MANETs that maximizes the network lifetime by finding routing solutions that minimize the variance of the remaining energies of the nodes in the network. Although this scheme introduces some additional traffic, simulations show that it improves the network lifetime by about $20-30 \% .^{1}$
\end{abstract}

\section{Introduction}

A mobile adhoc network is one where in all nodes work independent of any common centralized admin. Each one of them performs the tasks of a router. They should be self-adapting in that if their connection topology changes, their routing tables should reflect the change. Also, since they are mobile, they largely run on finite batteries. This means that they are power-constrained. Hence, it is an important design constraint for them to be poweraware or minimal in power expenditure. Also, each node should not be greedy about its own power since failure of some nodes in the network might result in lack of connectivity between nodes that are alive also.

Conventional MANET routing protocols do not consider power as a design constraint. Hence, they work towards optimal routes in terms of delay, which mostly results in the shortest path. This would mean that nodes with higher node degree might "die" soon since they are being used in most cases.

The paper is structured as follows:

Section 2 gives a brief classification of the broad domain of ad hoc routing protocols. Section 3 contains review of some research in low power ad hoc routing protocols.

Section 4 describes the rationale and details of the proposed Lifetime Prediction Routing (LPR) algorithm.

Section 5 elaborates on the simulation environment, the implementation and the experimental results comparing LPR with DSR, another popular ad hoc routing technique.

\section{MANET Routing Protocols}

Routing protocols in ad hoc networks are categorized in two groups: Proactive (Table Driven) and Reactive (On-Demand) routing.

\subsection{Proactive (Table-Driven) Routing Protocols}

These routing protocols are similar to and come as a natural extension of those for the wired networks. In proactive routing,

\footnotetext{
${ }^{1}$ Initial draft. Please do not distribute.
}

each node has one or more tables that contain the latest information of the routes to any node in the network. Each row has the next hop for reaching a node/subnet and the cost of this route. Various table-driven protocols differ in the way the information about a change in topology is propagated through all nodes in the network. Several proactive routing protocols are addressed in [1], [2] and [6].

\subsection{Reactive (On-Demand) Protocols}

Reactive routing is also known as on-demand routing. These protocols take a lazy approach to routing. They do not maintain or constantly update their route tables with the latest route topology. Examples of reactive routing protocols are the dynamic source Routing (DSR) [3][6], ad hoc on-demand distance vector routing (AODV) [4] and temporally ordered routing algorithm (TORA)[5].

Our power-aware source routing algorithm belongs to this category of routing algorithms. Since our approach is an enhancement over DSR, a brief description of DSR is warranted.

DSR is one of the more generally accepted reactive routing protocols. In DSR, when a node wishes to establish a route, it issues a route request (RREQ) to all of its neighbors. Each neighbor broadcasts this RREQ, adding its own address in the header of the packet. When the RREQ is received by the destination or by a node with a route to the destination, a route reply (RREP) is generated and sent back to the sender along with the addresses accumulated in the RREQ header. Since this process may consume a lot of bandwidth, DSR provides each node with a route cache to be used aggressively to reduce the number of control messages that must be sent. If a node has a cache entry for the destination, when a route request for that destination is received at the node, it will use the cached copy rather than forwarding the request to the network. In addition, each node promiscuously listens to other control messages (RREQs and RREPs) for additional routing data to add to its cache.

\section{Previous Work}

The main focus of research on routing protocols in MANETs has been network performance. There has been some study on poweraware routing protocols for MANETs. Presented below is a brief review of some of them.

\subsection{Power-aware Routing}

Reference [9] proposes a routing algorithm based on minimizing the amount of power (or energy per bit) required to get a packet from source to destination. More precisely, the problem is stated as:

$$
\operatorname{Min}_{\pi} \sum_{i \in \pi} T_{i, i+1}
$$

where $T_{i, i+1}$ denotes the power expended for transmitting (and receiving) between two consecutive nodes, $i$ and $i+1$ (a.k.a. link cost), in route $\pi$. 
This link cost can be defined for two cases:

- When the transmit power is fixed

- When the transmit power is varied dynamically as a function of the distance between the transmitter and intended receiver.

For the first case, energy for each operation (receive, transmit, broadcast, discard, etc.) on a packet is given by [7]:

$$
E(\text { packet })=b \times \text { packet_size }+c
$$

where $b$ and $c$ are the appropriate coefficients for each operation. Coefficient $b$ denotes the packet size-dependent energy consumption whereas $c$ is a fixed cost that accounts for acquiring the channel and for MAC layer control negotiation.

The second case is more involved. Reference [8] proposes a local routing algorithm for this case. The authors assume that the power needed for transmission and reception is a linear function of $d^{\alpha}$ where $d$ is distance between the two neighboring nodes and $\alpha$ is a parameter that depends on the physical environment. The authors make use of the GPS position information to transmit packets with the minimum required transmit energy. The key requirement of this technique is that the relative positions of nodes are known to all nodes in the MANET. However, this information may not be readily available. In addition, the GPS-based routing algorithm has two drawbacks. One is that GPS cannot provide nodes useful information about the physical environment (blockages, bit error rates, etc.) and the second is that the power dissipation overhead of the GPS device is an additional power draw on the battery source of the mobile node.

\subsection{Battery-cost Lifetime-aware Routing}

The main disadvantage of the problem formulation of reference [9] is that it always selects the least-power cost routes. As a result, nodes along these least-power cost routes tend to "die" soon because of the battery energy exhaustion. This is doubly harmful since the nodes that die early are precisely the ones that are needed most to maintain the network connectivity (and hence useful service life). Therefore, it will be better to use a higher power cost route if this routing solution avoids using nodes that have a small amount of remaining battery energy. This observation has given rise to a number of "battery-cost lifetime-aware routing" algorithms as described next.

1. Minimum battery cost routing algorithm minimizes the total cost of the route. More precisely, this algorithm minimizes the summation of inverse of remaining battery capacity for all nodes on the routing path [10].

2. Min-Max battery cost routing algorithm is a modification of the minimum battery cost routing. This algorithm attempts to avoid the route with nodes having the least battery capacity among all nodes in all possible routes. Thereby, it results in smooth use of the battery of each node [9][10].

3. Conditional Max-Min battery capacity routing algorithm was proposed in [10]. This algorithm chooses the route with minimal total transmission power if all nodes in the route have remaining battery capacities higher than a threshold; otherwise, routes that consist of nodes with the lowest remaining battery capacities are avoided. Several experiments have been performed in [10] to compare different battery cost-aware routing in terms of the network lifetime. According to their reported results, the minimum battery cost routing exhibited superior results compared to the Min-Max battery cost routing in terms of the expiration times of the nodes in the network. Conditional Min-Max routing showed better or worse results depending on how the threshold value was chosen.

4. Maximum Residual Packet Capacity (MRPC) was proposed in [14]. MRPC is conceptually similar to the conditional Min-Max battery cost, but MRPC identifies the capacity of a node not just by the residual battery capacity, but also by the expected energy spent in reliably forwarding a packet over a specific link.

5. Power-aware Source Routing (PSR) (proposed in [16]) is an on-demand source routing that uses state of the charge of battery to maximize the lifetime of a MANET. PSR solves the problem of finding a route $\pi$ at route discovery time $t$ as follows:

$$
\begin{aligned}
& \underset{\pi}{\operatorname{Min}} C(\pi, t)=\sum_{i \in \pi} C_{i}(t) \\
& \text { where } C_{i}(t)=\rho_{i} \cdot\left(\frac{F_{i}}{E_{r, i}(t)}\right)^{\alpha} \\
& \rho_{i}: \text { transmit power of node } \mathrm{i} \\
& F_{i}: \text { full-charge battery capacity of node } \mathrm{i} \\
& E_{r, i}(t) \text { : remaining battery cap. of node } \mathrm{i} \text { at time } \mathrm{t} \\
& \alpha: \text { a positive weighting factor }
\end{aligned}
$$

PSR use an accumulative graded cost function as described next. $\alpha$ is defined as a function of the ratio of the remaining battery capacity over the full-charge battery capacity. As this ratio decreases and becomes less than a specified set of threshold values, $\alpha$ increases according to a fixed schedule. In this way, nodes with very low battery capacity contribute a much higher value to the total path cost. In other words, if a path from source to destination has some nodes with a very low residual battery, the cost of the path will be very high, and therefore, PSR will behave similar to the MaxMin battery cost routing. We have compared the PSR and our proposed lifetime routing in terms of network lifetime in the experimental result section. Reference [13] uses a similar cost function to PSR in that its objective is to maximize the lifetime of a network with static or slowly varying topology by finding optimal traffic splits.

\section{Lifetime Prediction Routing}

\subsection{Basic Mechanism}

Lifetime Prediction Routing (LPR) is an on demand source routing protocol that uses battery lifetime prediction. The objective of this routing protocol is to extend the service life of MANET with dynamic topology. This protocol favors the path whose lifetime is maximum. We represent our objective function as follow:

$$
\underset{\pi}{\operatorname{Max}} T_{\pi}(t)=\underset{i \in \pi}{\operatorname{Min}}\left(T_{i}(t)\right)
$$

where: 
$\mathrm{T}_{\pi}(t)$ : lifetime of path $\pi$

$\mathrm{T}_{i}(\mathrm{t})$ : predicted lifetime of node $\mathrm{i}$ in path $\pi$

Lifetime Prediction: Each node tries to estimate its battery lifetime based on its past activity. This is achieved using a Simple Moving Average (SMA) predictor by keeping track of the last $N$ values of residual energy and the corresponding time instances for the last $\mathrm{N}$ packets received/relayed by each mobile node. This information is recorded and stored in each node. We have carefully compared the predicted lifetimes based on the SMA approach to the actual lifetimes for different values of $N$ and found $N=10$ to be a good value.

Our motivation in using lifetime prediction is that mobility introduces different dynamics into the network. In [13] the lifetime of a node is a function of residual energy in the node and energy to transmit a bit from the node to its neighbors. This metric works well for static networks for which it was proposed. However, it is very difficult to efficiently and reliably compute this metric when we have mobility since the location of the nodes and their neighbors constantly change.

PSR does not use prediction and only uses the remaining battery capacity. We believe LPR is superior to PSR since LPR not only captures the remaining (residual) battery capacity but also accounts for the rate of energy discharge. This makes the cost function of LPR more accurate as opposed to just using battery capacity. This is true in MANETs since mobility can change the traffic patterns through the node, which thereby affects the rate of depletion of its battery. Also, recent history is a good indicator of the traffic through the node and hence we chose to employ lifetime prediction.

Our approach is a dynamic distributed load balancing approach that avoids power-congested nodes and chooses paths that are lightly loaded. This helps LPR achieve minimum variance in energy levels of different nodes in the network. As an example, consider the scenario shown in figure 1 . Here, node $\mathrm{F}$ has two flows going through it $(\mathrm{D} \rightarrow \mathrm{F} \rightarrow, \mathrm{B} \rightarrow \mathrm{F} \rightarrow$ and $\mathrm{C} \rightarrow \mathrm{F} \rightarrow)$. Now, if $\mathrm{A}$ wants to transmit data to $\mathrm{E}$, the shortest path routing will use $\mathrm{A} \rightarrow \mathrm{F} \rightarrow \mathrm{E}$. However, LPR will use $\mathrm{A} \rightarrow \mathrm{B} \rightarrow \mathrm{C} \rightarrow \mathrm{D} \rightarrow$ E since $\mathrm{E}$ is very power-congested (as a result of relaying multiple flows) and the path passing through $\mathrm{F}$ will not be selected by LPR.

\subsection{Route Discovery}

In DSR, activity begins with the source node flooding the network with RREQ packets when it has data to send. An intermediate node broadcasts the RREQ unless:

- It gets a path to the destination from its cache, or

- It has previously broadcast the same RREQ packet. (this fact is known from the sequence number of the RREQ and the sender ID.)

Consequently, intermediate nodes forward only the first received RREQ packet. The destination node only replies to the first arrived RREQ since that packet tends to take the shortest path.

In LPR, all nodes except the destination calculate their predicted lifetime, $T_{i}$ (cf. Equation 6 ) and replace the min lifetime in the header with $T_{i}$ if $T_{i}$ is lower than the existing min lifetime value in the header.

$$
T_{i}(t)=\frac{E_{r, i}(t)}{\frac{1}{N-1} \sum_{k=i-N+1}^{i} R_{k}(t)}
$$

where:

$E_{r, i}(t)$ : remaining energy at the $\mathrm{i}^{\text {th }}$ packet is being sent or relayed through the current node

$R_{k}(t)$ : rate of energy depletion of the current node when the $\mathrm{k}^{\text {th }}$ packet was sent and is calculated by as the ratio of the difference between residual energies of the nodes for packets $k-1$ and $k$ and the difference between arrival times of these two packets.

$N$ : length of the history used for calculating the SMA

When an intermediate node receives a RREQ packet, it starts a timer $\left(T_{r}\right)$ and keeps the min. lifetime in the header of that packet as Min-Lifetime. If additional RREQs arrive with same destination and sequence number, the cost of the newly arrived RREQ packet is compared to the Min-Cost. If the new packet has a lower cost, Min-Cost is changed to this new value and the new RREQ packet is forwarded. Otherwise, the new RREQ packet is dropped (Table $1)$.

Table 1: Pseudocode of functionality in intermediate node

\author{
Intermediate Node: \\ Predict Its lifetime, \\ If its lifetime $<$ Min-lifetime \\ Replace Min-lifetime with its lifetime \\ If Sequence Number exists \\ Compare Min-lifetime of current RREQ with Min- \\ lifetime of existing one. \\ If new Min-lifetime $<=$ old Min-lifetime \\ Discard new RREQ \\ If new Min-Lifetime >old Min-lifetime \\ Replace old Min-Lifetime with new Min- \\ lifetime \\ Forward new RREQ \\ If Sequence Number does not exist \\ Save this Min-lifetime \\ Forward RREQ
}

In LPR, the destination waits for a threshold number $\left(T_{r}\right)$ of seconds after the first RREQ packet arrives. During that time, the destination examines the cost of the route of every arrived RREQ packet. When the timer $\left(T_{r}\right)$ expires, the destination node selects the route with the minimum cost and replies. Subsequently, it will drop any received RREQs. The reply also contains the cost of the selected path appended to it. Every node that hears this route reply adds this route along with its cost to its route cache table. Although this scheme can somewhat increase the latency of the data transfer, it results in a significant power saving as will be shown later. A simple example of this process is illustrated in figure 2. Here, the route A-B-C-D is chosen by LPR over the route A-E-D since the path lifetime of the former is $500 \mathrm{~s}$, which is greater than the latter.

LPR has a route invalidation timer that invalidates old routes. This helps in removing old routes. This also avoids over usage of particular routes in cases of low mobility. 


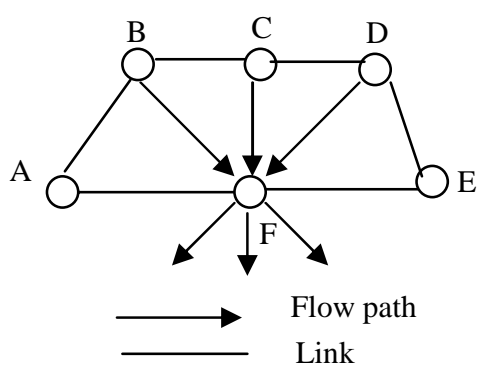

Figure 1: LPR avoids power-congested paths

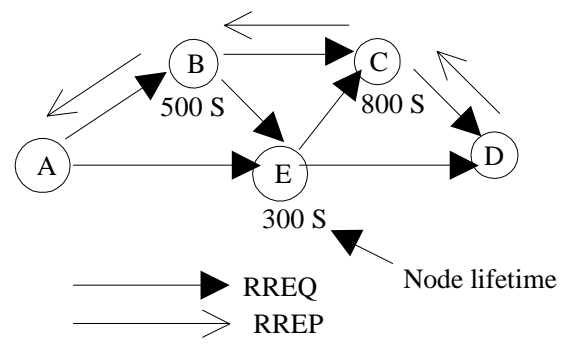

Figure 2:Route setup process in LPR

\subsection{Route Expiration}

Route maintenance is needed for two reasons:

- Mobility: Connections between some nodes on the path are lost due to their movement,

- Change in predicted lifetime

In the first case, a new RREQ is sent out and the entry in the route cache corresponding to the node that has moved out of range is purged. Following policy is adopted to tackle the second situation: Once the route is established, the weakest node in the path (the node with minimum predicted lifetime at path discover time) monitors the decrease in its battery lifetime. When this remaining lifetime decrease goes beyond a threshold level, the node sends a route error back to the destination as if the route was rendered invalid. The destination sends this route error message to the source. This route error message forces the source to initiate route discovery again. This decision is only dependent on the remaining battery capacity of the current node and its discharge rate in the short history. Hence is a local decision. LPR adopts this local approach because this approach minimizes control traffic. Figure 3 shows an example of route expiration process.

More precisely, node $i$ generates a route error at time $t$ when the following condition is met:

$$
\begin{aligned}
& \mathrm{T}_{\mathrm{i}}\left(\mathrm{t}_{0}\right)-\mathrm{T}_{\mathrm{i}}(\mathrm{t}) \geq \delta \\
& \text { where } \\
& \mathrm{T}_{\mathrm{i}}: \text { predicted lifetime of node } \mathrm{i} \\
& \mathrm{t}: \text { current time } \\
& \mathrm{t}_{0}: \text { route discovery time } \\
& \delta: \text { change threshold for lifetime prediction }
\end{aligned}
$$
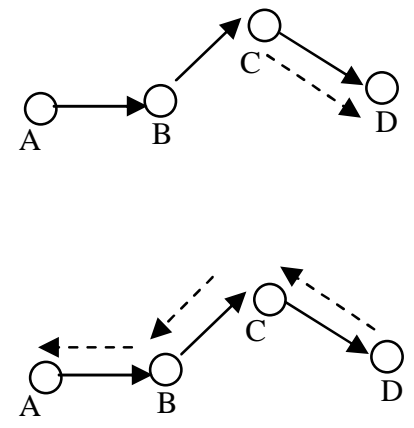

(b)

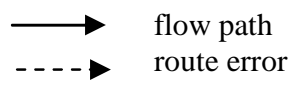

Figure 3: (a) $\mathrm{C}$ sends route error to destination (D) (b) send route error to the source (A)

\section{Experimental Results}

\subsection{Simulation Setup}

We used the event driven simulator ns-2 [11] along with the wireless extensions provided by CMU [12]. The simulation consists of a network of 20 nodes confined in a $1000 \times 1000 \mathrm{~m}^{2}$ area. Random connections were established using CBR traffic (at 4 packets/second) such that each node has chance to connect to every other node. Packet size was 512 bytes and each simulation was executed for $20000 \mathrm{sec}$. The initial battery capacity of each node is 100 units. Nodes followed random waypoint mobility model with a specific max velocity and no pause time. Each packet relayed or transmitted consumes a fixed amount of energy from the battery as given by equation (2). a and $\mathrm{b}$ are constants.

The key parameters of study are the network lifetime, node lifetime and RMS of energy consumption $\left(E_{R M S}\right)$ in the network. We vary the speed and radio transmission range and study their effects on these metrics.

\subsection{Simulation Results}

The network lifetime is defined as the time taken for a fixed percentage of the nodes to die due to energy resource exhaustion. Network lifetime of DSR, PSR and LPR are compared for a given scenario. Here, the speed of each node is $10 \mathrm{~m} / \mathrm{s}$ and radio transmission range is $125 \mathrm{~m}$. Figure 4 shows the time instances at which a certain number of nodes have died when simulating LPR, PSR and DSR. Note that in figure 4 the node death of all 20 nodes is not shown since some nodes are still alive at the end of the simulation. Some of these nodes are however rendered unreachable since many of the nodes have exhausted their energy and hence they cannot reach other nodes consistently.

As can be seen, the first node in DSR and PSR dies about $20 \%$ earlier than in the case of LPR. Similarly, in DSR 5 nodes die approximately $32 \%$ earlier than LPR and $27 \%$ earlier than LPR in the case of PSR.

To increase the lifetime of the network, the variance of the residual energy of the nodes should be minimized. Figure 4 is not very informative in this regard. A histogram of the snapshots of the energy consumption in each of the nodes at different time instances would be more informative. One of the ways to compare such histograms would be to look at the RMS of remaining energy 
$\left(E_{R M S}\right)$ at different time instants ([15]). It provides information about the total energy consumed and spread of consumed (or residual) energy. Figure 5 shows the evaluation of ERMS as a function of time for DSR and LPR. The effect of mobility on $E_{R M S}$ can also be seen in this figure. A linear estimation of $E_{R M S}$ is shown for ease of comparison. As can be seen LPR is always better than DSR in terms of ERMS value. This graph is in agreement with our expectations. However, as the velocity of node movement increases, rate of energy consumption in the network goes up. This is expected since higher velocity of movement implies more route discoveries being performed and as a consequence higher energy consumption in the network. Also, as the node mobility increases, the difference between DSR and LPR decreases. This could be attributed to two reasons:

- $\quad$ LPR makes use of the fact that DSR overloads certain nodes and has a big variance between remaining energies of the nodes. As mobility increases, the amount of overhead (control packets for route discovery) increases for both DSR and LPR. As a consequence, there is less room for LPR to balance the energy consumption among the nodes in the network and extend its network lifetime.

- Because there are more route discoveries, no paths are overused even by DSR. As a consequence, DSR also achieves load balancing to an extent decreasing the gain seen by LPR.

Packet delivery ratio is defined as the number of delivered data packets to the number of generated data packets in all nodes. Note that the number of generated packets is the "expected" number of generated packets. We generate as many as 200,000 data packets during the simulation. They are generated between random source and destination pairs at random times. Many of these might not have reached their intended destination because of lack of existence of a route between the source and destination for various reasons. Also, the network lifetime clearly affects this ratio. If the network was alive for longer time, it implies that more data traffic goes through since we establish random connections throughout the time of the simulation.

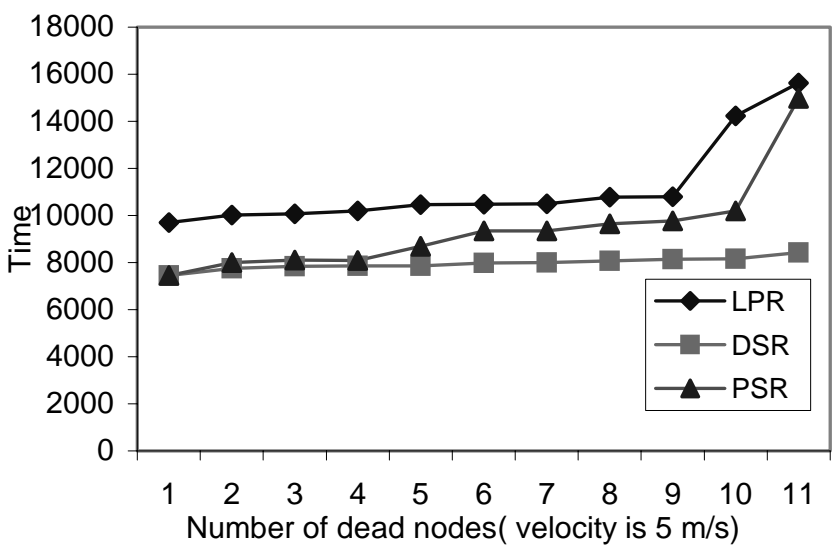

Figure 4: The number of dead nodes in DSR, PSR and LPR versus time

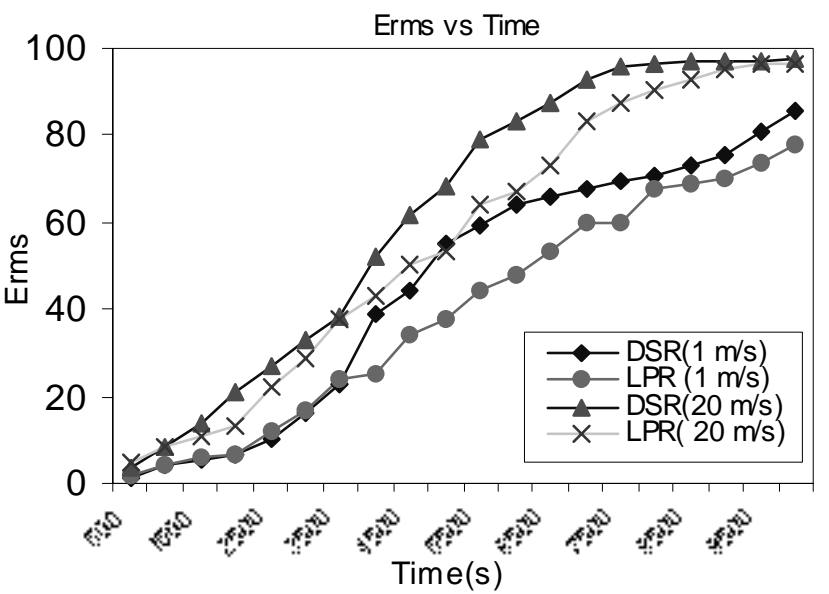

Figure 5:Evaluation of ERMS for different velocities of node movement

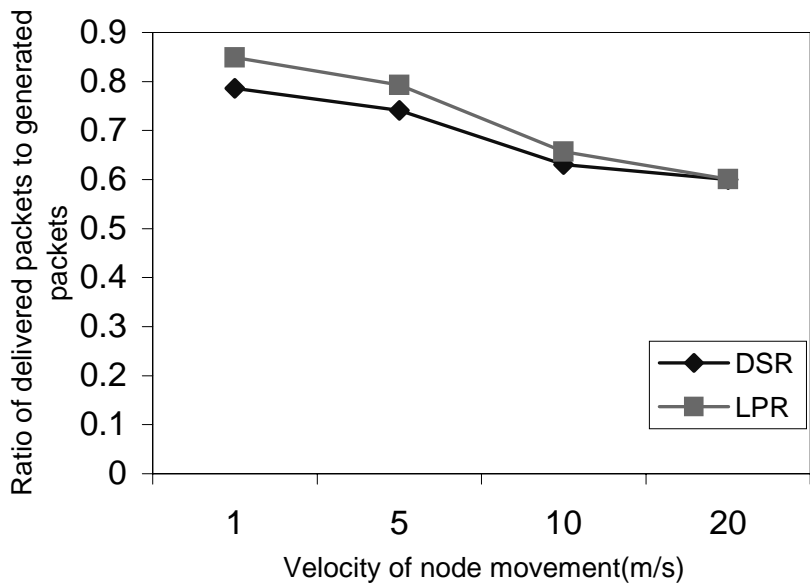

Figure 6: Packet delivery ratio vs. Velocity of node movement

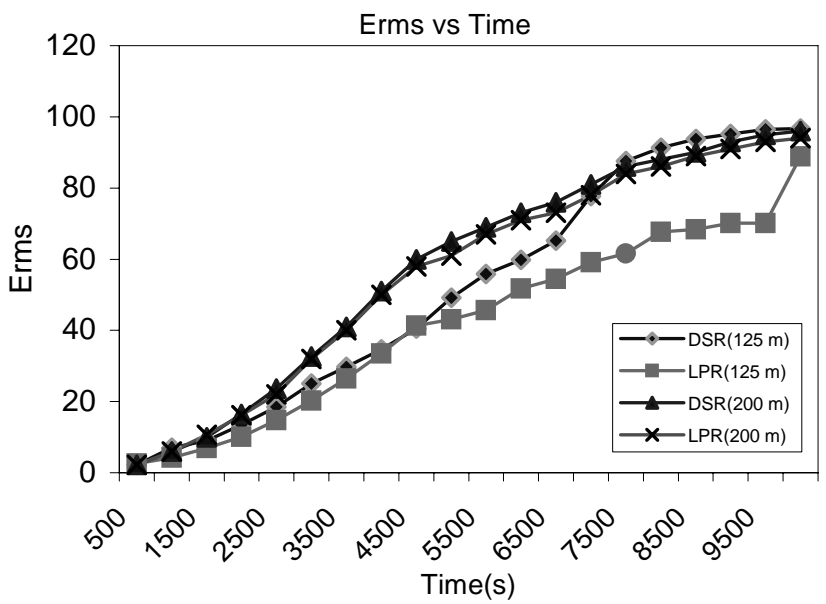

Figure 7: Effect of transmission range on the evaluation of $E_{R M S}$ (velocity is $5 \mathrm{~m} / \mathrm{s}$ )

In figure 6 we can see that for lower velocities of node movement, LPR has a greater ratio of delivered packets. However, 
as the mobility increases, this ratio goes down. The intuition for why LPR does not perform as well in higher velocities has been presented above.

The transmission range is another parameter that can affect the performance of routing protocols because it changes the connectivity of the network. We changed the transmission range to see the effect of the degree of connectivity on our metric (cf. Figure 7). We assume same transmission power for all nodes in a simulation. The node transmit range was assigned two different values $(125,200 \mathrm{~m})$ for the simulations. We make the following observations based on this figure:

- When the transmission range increases, each node covers more nodes. In other words, when a node sends unicast or broadcast packet more nodes will receive packets and they consume power in their receiver part. Hence, each transmission has lot of power overhead for the network. As a result when range increases nodes discharge faster.

- The number of hops per route decreases by increasing the transmission range. Hence, nodes have less participation in relaying packets resulting in lower activity for each node and slower discharge of its battery capacity.

When range increases from 125 to 200 , the dominant effect is the first one and charge rate of the nodes increase drastically. Both of those effects reduce the effect of the LPR scheme and as can be seen the difference between LPR and DSR decreases such that when the range is 200 the difference is not clear. To reduce the cost of the power due to the second effect, one way is to shut down the non-destined nodes in the range of a transmitting node

In LPR, route discovery process needs more control packets to be propagated in the network since it needs to compare all possible paths between a source and a sink and selects a path with maximum lifetime. To show the overhead of LPR on the network we have measured the ratio of number of control packets to number of delivered packets in the network. This normalizes the overhead of the routing protocol to the goodput (number of received packets) in the network. Figure 8 shows this ratio for LPR and DSR for different velocities of node movement and for 380 UDP connections. As the velocity of movement increases, routes are valid for shorter time and more route discoveries are done in the network resulting in more control packets and more the difference between LPR and DSR. LPR increases the ratio of control packet to transmit packet less than $4 \%$. The increase in the size of a control packet in DSR to that in LPR is approximately $1 / 10$ and the overhead in energy for sending such a packet increases by approximately $0.4 \%$. Hence, the additional energy overhead of LPR for route discovery is small.

\section{Conclusion}

In this paper, we elaborate on Lifetime Prediction Routing (LPR), a routing protocol to enhance the lifetime of a given mobile adhoc network. We compare it with dynamic source routing (DSR), a popular routing technique used in MANETs which does not consider power but optimizes routing for shortest delay. The main objective of LPR is to minimize the variance in the remaining energies of all the nodes and thereby prolong the network lifetime. It achieves this by doing local decisions and with minimum overhead. We show that LPR brings about a clear increase in network lifetime. Finally, we also point out some of the overheads of using LPR and show clearly when LPR performs better and what are the factors that affect it.

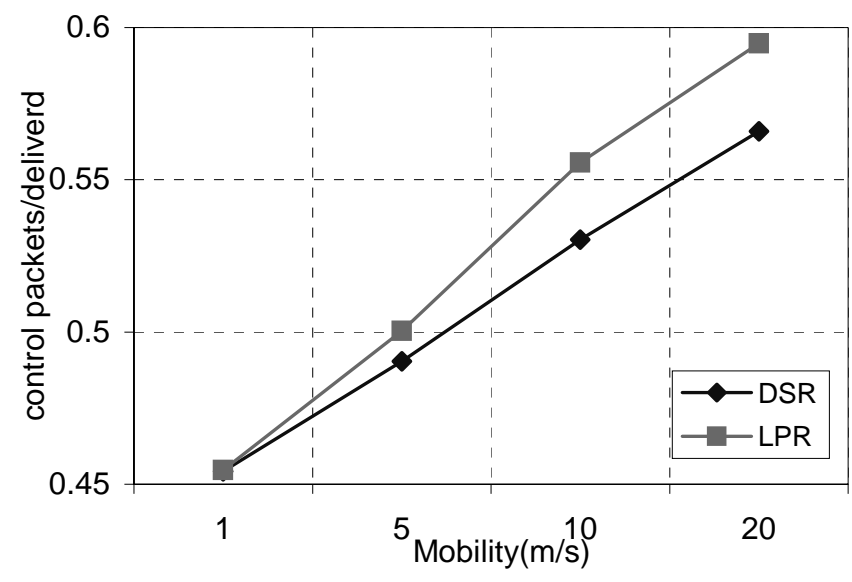

Figure 8: The ratio of control packets to delivered packets as a function of velocity of node movement for LPR and DSR for 380 UDP connections

\section{References}

1. C. Perkins and P. Bhagwat. "Highly Dynamic Destination-Sequenced Distance-Vector Routing (DSDV) for Mobile Computers". Proc. of ACM SIGCOMM, October 1994.

2. Murthy and J.J. Garcia-Luna-Aceves, "An Efficient Routing Protocol for Wireless Networks," ACM Mobile Networks and Applications Journal, Special issue on Routing in Mobile Communication Networks, Vol. 1, No. 2, 1996.

3. David B. Johnson, David A. Maltz, Yih-Chun Hu and Jorjeta G. Jetcheva. "The Dynamic Source Routing for Mobile Ad Hoc Wireless Networks," http://www.ietf.org/internet-drafts/draft-ietfmanet-dsr-06.txt, IETF Internet Draft, Nov. 2001, Work in progress.

4. Charles E. Perkins, Elizabeth M. Belding-Royer, and Samir Das. "Ad Hoc On Demand Distance Vector (AODV) Routing." IETF Interne12t draft, draft-ietf-manet-aodv-09.txt, November 2001 (Work in Progress).

5. V.Park and S.Corson. "Temporally-Ordered Routing Algorithm (TORA) Version 1", IETF Internet Draft, July 2001, (Work in progress).

6. Charles E.Perkins, "Ad hoc Networking", Addison Wesley, 2001

7. S. Lindsey, K. Sivalingam and C.S. Raghavendra, " Power Aware Routing and MAC protocols for Wireless and Mobile Networks", in Wiley Handbook on Wireless Networks and Mobile Computing; Ivan Stojmenivic, Ed., John Wiley \& Sons, 2001

8. Stojmenovic and X. Lin, "Power-aware localized routing in wireless networks." Proc. IEEE IPDPS, Cancun, Mexico, May 2000.

9. S. Singh , M.Woo and C.S. Raghavendra, "Power-Aware Routing in Mobile Ad hoc Networks," Proceedings of Mobicom 98.

10. C.K. Toh, "Maximum Battery Life Routing to Support Ubiquitous Mobile Computing in Wireless Ad hoc Networks", IEEE Communication Magazine, June 2001.

11. ns-2 Manual.http://www.isi.edu/nsnam/ns/doc/index.html

12. CMU Monarch Extensions to ns. http://www.monarch.cs.rice.edu/

13. J-H Chang and L. Tassiulas, "Energy Conserving Routing in Wireless Ad-hoc networks", Proceedings of INFOCOM 2001.

14. A. Misra and S.Banerjee, "MRPC: Maximizing Network Lifetime for reliable routing in Wireless Environments", IEEE Wireless Communications and Networking Conference, 2002.

15. C. Schurgers and M.B. Srivastava, "Energy efficient routing in wireless sensor networks", IEEE Wireless Communications and Networking Conference, 2002.

16. M.Maleki, K. Dantu and M. Pedram, " Power -aware Source Routing in mobile ad hoc networks", Proceedings of ISLPED '02, Monterey, $C A$. 